\title{
SEGREGACIÓN SOCIAL EN LAS ESCUELAS PÚBLICAS Y PRIVADAS EN AMÉRICA LATINA*
}

\author{
F. JaVIER MURILLO ${ }^{1}$ \\ Cynthia Martínez Garrido ${ }^{2}$
}

\begin{abstract}
RESUMEN: Esta investigación estudia la relación entre educación privada y segregación escolar por razón socioeconómica en América Latina. Para ello, realiza una explotación especial de la base de datos del TERCE estimando los índices de Disimilitud, Inclusión Socioeconómica y Raíz Cuadrada a una muestra de 119.967 estudiantes de 5.733 escuelas de quince países de América Latina. Los resultados indican que: (i) existe una fuerte relación positiva entre el peso de la educación privada y la segregación de un país, y (ii) las escuelas privadas segregan más que las escuelas públicas, especialmente a los estudiantes con familias de menor nivel socioeconómico; sin embargo, hay muchas diferencias por países. Con ello se alerta de los efectos negativos sobre la equidad educativa de las políticas de fomento de la educación privada en América Latina.
\end{abstract}

Palabras clave: Segregación escolar. Escuelas públicas y privadas. Nivel socioeconómico. América Latina.

\section{SOCIAL SEGREgATION IN PUBLIC AND PRIVATE SCHOOLS IN LATIN AMERICA}

ABSTRACT: This research studies the relationship between private education and school segregation by socioeconomic status in Latin America. To achieve our aim, we conducted a special data mining of the database of TERCE estimating the index of Dissimilarity, SocioEconomic Inclusion, and Square Root of a sample of 119,967 students from 5,733 schools in 15 in Latin American countries. The results indicate that: (i) there is a strong positive relationship between the weight of private education and segregation index of a country, and (ii) private schools are more segregated than public schools, especially students with less socioeconomic status families; however, there are many differences between countries. Our research warns about the

\footnotetext{
*Este artículo ha sido desarrollado en el marco del Proyecto I+D+i de Excelencia "Escuelas en contextos socioeconómicamente desafiantes: Una aproximación desde la Educación para la Justicia Social de Investigación" Ref: EDU2014-56118-P. Financiado con fondos del Ministerio de Economía y Competitividad, Gobierno de España.

${ }^{1}$ Universidad Autónoma de Madrid - Madrid, España. E-mail: javier.murillo@uam.es

${ }^{2}$ Universidad de Zaragoza - Zaragoza, España. E-mail: cynthia@unizar.es

DOI: 10.1590/ES0101-73302017167714
} 
negative effects on educational equity policies encouraging of private education in Latin America.

Keywords: School segregation. Public and private schools. Socioeconomic status. Latin America.

\title{
LA SÉGRÉGATION SOCIALE DANS LES ÉCOLES PUBLIQUES ET PRIVÉES EN AMÉRIQUE LATINE
}

\begin{abstract}
$R E ́ S U M E ́$ : Cette recherche étudie la relation entre l'enseignement privé et la ségrégation scolaire en raison de la condition socio-économique en Amérique latine. Pour cela, on réalise une exploration spéciale de la base de données de TERCE en estimant les indices de "dissemblance ", " inclusion socio-économique " et " racine carrée " d'un échantillon de 119.967 élèves de 5.733 écoles dans 15 pays de l'Amérique Latine. Les résultats indiquent que : (i) il y a une forte relation positive entre le poids de l'enseignement privé et l'indice de ségrégation d'un pays, et (ii) les écoles privées font plus de ségrégation que les écoles publiques, en particulier des étudiants provenant de familles de statut socioéconomique plus bas ; cependant, il y a de nombreuses différences entre les pays. Notre recherche met en garde contre les effets négatifs sur les politiques d'équité éducatives encourageant l'enseignement privé en Amérique Latine.
\end{abstract}

Mots-clés : Ségrégation scolaire. Écoles publiques et privées. Statut socioéconomique. Amérique Latine.

\section{Introducción}

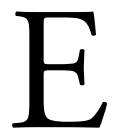

$s$ posible afirmar sin temor a equivocarse que uno de los mayores desafíos de la Educación en América Latina es la inequidad de sus sistemas educativos. Efectivamente, haciendo buena la máxima de que "la educación reproduce y legitima las diferencias en la sociedad" (BOURDIEU; PASSERON, 1977), las sociedades más inequitativas del mundo solo pueden generar y estar generados por sistemas educativos de análogas características.

Uno de los indicadores de inequidad es la llamada segregación escolar, término que hace referencia a la distribución desigual de los estudiantes en los centros educativos según sus características personales o sociales (p.ej., ALLEN; VIGNOLES, 2007; DUPRIEZ, 2010; FRANKEL; VOLIJ, 2011). Esas características pueden ser el país de nacimiento, la cultura, el género, la capacidad o el nivel socioeconómico de las familias de los estudiantes. Este trabajo aborda la segregación socioeconómica por ser la que está más directamente ligada a la inequidad social. 
De esta forma, el fenómeno de la segregación socioeconómica de las escuelas indica que los niños, niñas y adolescentes más cuyas familias tienen menores recursos se concentran en algunas escuelas mientras que en otras se agrupan los hijos e hijas de las familias con mayores posibilidades socioeconómicas.

En esta investigación nos interesa relacionar la segregación escolar con el incremento de la Educación Privada, fenómeno que se está dando en todo el mundo pero con especial intensidad en América Latina. El imaginario social está instalado en la idea de que los centros privados ofrecen una educación de mayor calidad que los de titularidad pública. Sin entrar en su discutible veracidad, quedemos dar un paso más allá y abordar el papel de la educación privada en la construcción de sistemas educativos más equitativos. Concretamente nos preguntamos, ¿la educación privada está contribuyendo a una mayor segregación escolar o a su disminución?

Teniendo en cuenta que, más allá de presiones sociales, el incremento de la educación privada en un país es producto de políticas gubernamentales más o menos explicitas, abordar este tema es una forma de contribuir a trabajar por una menor segregación que, a la postre, contribuye a una sociedad menos inequitativa y más justa.

Con esta investigación se pretende alcanzar dos objetivos. Por un lado, se busca explorar la relación entre educación privada y segregación escolar por razón socioeconómica en los países de América Latina, y, por otro, determinar la magnitud segregación de los subsistemas de educación pública y privada en cada país.

\section{Marco teórico}

\section{Segregación escolar}

Entendemos segregación como la distribución desigual de los individuos en diferentes estructuras jerárquicas según sus características (JAMES; TAEUBER, 1982). Aplicado a la educación, segregación escolar es el agrupamiento de algunos estudiantes en determinados centros educativos en función de sus características personales o sociales. Optamos, por tanto, por una concepción de segregación escolar centrada en la dimensión de igualdad (evenness) (GORARD; TAYLOR, 2002; MASSEY; DENTON, 1988).

En función de la característica social o personal de los estudiantes que genera su segregación es posible hablar de segregación escolar étnico-racial, cuando estudiantes de una determinada minoría étnica se concentran en unos pocos centros, de segregación escolar por origen, cuando la concentración de estudiantes inmigrante extranjeros en unas escuelas, de segregación por capacidad, o incluso de segregación por género. En este estudio hemos optado por trabajar con la se- 
gregación por nivel socioeconómico por su influencia en las inequidades sociales. Según la misma, existe una distribución desigual de los estudiantes en las escuelas en función del nivel socioeconómico y cultural de sus familias.

El interés por la segregación escolar nació como consecuencia del fallo de la Corte Suprema en 1954 que declaró inconstitucionales las leyes estatales que establecían escuelas públicas separadas para estudiantes de raza negra y blanca en el llamado caso Brown contra el Consejo de Educación de Topeka (COLEMAN, 1975). De esta forma, en los primeros momentos la investigación se centró en la segregación étnico-racial. Sin embargo, no fue hasta la década de los 80 cuando surgen los estudios sobre segregación escolar de carácter socioeconómico. Uno de los trabajos más influyentes fue el realizado por Wilson (1987) en el que se estudian las desventajas de la concentración de familias de clases sociales más desfavorecidas en algunas escuelas. Desde entonces se han multiplicado los trabajos que abordan la segregación escolar por nivel socioeconómico entre escuelas, su evolución, o su comparación entre diferentes países (p.e., DUPRIEZ, 2010; GORARD; SMITH, 2004; ORFIEL; LEE, 2005; STEPHAN, 2013).

En América Latina, la segregación escolar de carácter socioeconómico ha sido abordada de manera incipiente. Destacan algunos pocos trabajos como los elaborados por Elacqua (2012) y Valenzuela, Bellei y De los Ríos $(2010 ; 2014)$ en Chile, por Gasparini y otros (2011), Jaume (2013) y Krüger (2011; 2014) en Argentina, por Balarin (2016) y Benavides, León y Etesse (2014) en Perú, por Rangel (2006) y Rangel y Lleras (2010) en Colombia, o por Cárdenas Denham (2011) y Martínez Casas y Muller (2006) en México, por poner algunos ejemplos realizados en países concretos o por Mardones (2006), Vázquez (2012), Murillo (2016) y Murillo y Martínez-Garrido (2017a) con varios países de la Región. Todos ellos encuentran que América Latina tiene sistemas educativos altamente segregados.

Efectivamente, el estudio realizado por Vázquez (2012) con datos de PISA-2009 demuestra que el índice de Disimilitud entre los países participantes en el estudio varía entre 0,53 y 0,26. Siendo en América Latina donde se encuentran los países en los que se da una mayor segregación escolar entre los estudiados (Perú 0,53; Chile 0,52; México 0,49; Argentina 0,46; Colombia 0,46; Uruguay 0,44; y Brasil 0,42). Murillo (2016), por su parte, encuentra que la segregación escolar por nivel socioeconómico en los países de América Latina varía entre 0,48 en Uruguay y 0,66 en Panamá para el 25\% de los sujetos con menor ISEC, y de 0,54 en República Dominicana y 0,77 en Honduras para el 25\% de los sujetos con mayor ISECF (valores medidos a través del índice de Disimilitud).

Uno de los temas clave en la estimación de la magnitud de la segregación es elegir el índice más adecuado. Para calcular la segregación escolar desde la dimensión de la igualdad, por la que optamos, los índices más habituales son el índice de Disimilitud (DUNCAN; DUNCAN, 1955), el índice de Gorard (GORARD; TAYLOR, 2002), el índice de la Raíz Cuadrada (HUTCHENS, 2004) y el índice 
de Brecha por Centiles (WATSON, 2009). Pero también el menos habitual índice de Inclusión Socioeconómica a partir de Modelos Multinivel (MURILLO, 2016).

Para desarrollar una visión completa y robusta del estado de la segregación por nivel socioeconómico en las escuelas latinoamericanas en esta investigación se van a utilizar tres índices. Por una parte, el índice de Disimilitud, por ser el más fácil de interpretar y el más popular entre los investigadores, lo que nos permite comparar y discutir nuestros resultados con otros trabajos. Por otra el índice de Inclusión Socioeconómica, que supera alguna limitación del índice D; y, por último índice de Raíz cuadrada, dado que es el único que permite descomponer la segregación entre subsistemas, en este caso, educación pública y privada. En el apartado de metodología desarrollaremos en profundidad estos índices y abordaremos concretamente sus aportaciones individuales, y también sus limitaciones que justifican su uso combinado.

\section{Evolución de la educación privada}

Desde los ańos 90 hasta la actualidad, ha crecido de forma importante el número de escuelas privadas y, con ello, el porcentaje de niños, niñas y adolescentes escolarizadas en ellas en todo el mundo, tanto en países en desarrollo como en los desarrollados (WINKLER, 1988). Esencialmente son tres las razones que motivan el aumento de las escuelas privadas. En primer lugar, la falta de capacidad de las instituciones públicas para acoger al alumnado de la zona. En segundo término, la aparición de una demanda diferenciada de necesidades formativas propias de grupos específicos, bien sea por buscar una educación religiosa o la enseñanza de idiomas; en América Latina el crecimiento de una clase media con más recursos y que intenta diferenciarse entraña políticas gubernamentales que potencian la educación privada bien sea con la excusa de favorecer la libertad de elección de centro basadas en generación de cuasi-mercados educativos, o bien sea argumentando la supuesta mayor calidad de la educación privada, especialmente otorgando subvenciones y recursos públicos para su financiamiento (ALEGRE CANOSA, 2010).

En los países de América Latina este crecimiento ha sido especialmente alto. Así, según Arcidiácono y sus colaboradores (2014), los países latinoamericanos cuentan con un mayor porcentaje de estudiantes que acuden a escuelas privadas en comparación con el resto de países de la OCDE participantes en el estudio, y esta diferencia sigue creciendo. Concretamente, en el año 2000, el porcentaje de estudiantes que asisten a escuelas privadas en América Latina y el Caribe era del $14 \%$, frente al $10 \%$ que lo hacía en los países desarrollados. Una década más tarde, estas diferencias se agudizan. En el año 2010, el 11\% de los estudiantes acude a escuelas privadas en los países desarrollados; este porcentaje asciende hasta el 17\% en la Región latinoamericana. 
No todos los países de América Latina y el Caribe se han visto igual de afectados. Chile es el país que presenta un mayor incremento de la matrícula privada, pasó de tener el 22\% en 1981, al 45\% en 2006 y en 2010 ya alcanzó la cifra del 58\%. En segundo lugar se encuentran Perú (23\%) y República Dominicana (24\%), ambos con un aumento de 9 puntos porcentuales entre los años 2000 y 2010. De manera opuesta, El Salvador es el único país que presenta una disminución de la matrícula primaria privada, aproximadamente 5 puntos porcentuales desde el año 2000 a 2010. Por último, en Brasil y Uruguay hubo una disminución del porcentaje de alumnos que asisten a escuelas privadas entre 1990 y 2000, pero a partir del 2000 la tendencia se invirtió y en 2010 cuentan con los mimos valores que en 1990 ( $14 \%$ en Brasil y 17\% en Uruguay). Frente a estos casos, México destaca por contar con un $90 \%$ de sus estudiantes matriculados en escuelas públicas de Etapa Primaria (ARCIDIÁCONO et al., 2014; BELLEI, 2007; PEREYRA, 2008).

\section{Educación privada y sus efectos}

En estos años se han desarrollado un buen número de estudios que analizan el efecto de la titularidad de las escuelas sobre el rendimiento de los estudiantes. Las evidencias encontradas para América Latina, lejos de mostrar una imagen uniforme, reflejan la complejidad del fenómeno (ARISTIZABAL; GIMÉNEZ; XIMÉNEZ-DE-EMBÚN, 2016). De un lado, están los estudios que hallan que la escolarización en centros privados y su mayor autonomía mejora el rendimiento de los estudiantes en áreas como Lectura y Matemáticas (FERNÁNDEZ; DEL VALLE, 2013; GAMBOA; WALTENBERG, 2012). Estos sostienen sus planteamientos en evidencias como las aportadas por el SIMCE que destaca que desde los ańos 90 los estudiantes que asisten a escuelas privadas superan sistemáticamente a los alumnos de escuelas públicas, concretamente una diferencia en el rendimiento de entre 0,3-0,4 desviaciones estándar de puntajes SIMCE (BELLEI, 2007).

Sin embargo también hay un grupo de investigaciones que no encuentran relación alguna entre la titularidad del centro y el desempeño (DUARTE; BOS; MORENO, 2010; OCDE, 2011). Estos trabajos aluden al denominado sesgo de selección de las escuelas generado por tres razones: la oferta de escuelas privadas no se distribuye aleatoriamente entre las diferentes áreas geográficas y clases sociales; la disposición de las familias para enviar a sus hijos a las escuelas privadas varía de acuerdo a características familiares y de los alumnos, no todas ellas conocidas; y los extremadamente desregulados y desconocidos procesos de selección de los estudiantes por parte de los establecimientos, hacen que estos puedan aplicar variados criterios para discriminar a los postulantes, basándose en los intereses de la propia escuela. Un grupo más de estudios presentan resultados con un mayor grado de matización: la escolarización en centros privados favorece 
el desempeño en los estudiantes de países desarrollados y con alto rendimiento, pero afecta de manera negativa a los de países en vías de desarrollo y con bajo desempeño (HANUSHEK; WOESSMANN, 2012).

Sin embargo, mirar el sistema educativo exclusivamente bajo el prisma del rendimiento de los estudiantes es solo ver una parte de la realidad. Interesa también conocer hasta qué punto la educación privada está contribuyendo a una mayor equidad de los sistemas educativos o, por el contrario, está contribuyendo a potenciar las desigualdades sociales.

En coherencia con esa preocupación, existe una fructífera línea de trabajo que ha abordado esa temática (p.ej., ARCIDIÁCONO et al., 2014; BETTS; FAIRLIE, 2001; RUMBERGER; PARLARDY, 2005). Entre ellos destaca el seminal estudio de Coleman, Hoffer y Kilgore (1982) donde se abordó el tema de la equidad y la segregación en las escuelas privadas de los Estados Unidos. Sus resultados fueron concluyentes: el nivel socioeconómico de los estudiantes es hasta tres veces más alto en las escuelas privadas que en las públicas, lo que genera además poco contacto que entre estudiantes de diferente nivel socioeconómico. Así, hallaron que la proporción de estudiantes con alto nivel socioeconómico cuyos compañeros de clase tienen bajo nivel socioeconómico es de 0,14 en las escuelas públicas, $\mathrm{y}$ de 0,07 en las privadas.

Centrándonos en América Latina, merece ser destacado el estudio de Ana Pereyra (2008) para el SITEAL. En el mismo estudia la relación entre el nivel de ingresos de las familias y el tipo escolarización preferido para sus hijos. Los resultados con datos de 12 países de la región indican que el $9 \%$ de los estudiantes con menores recursos económicos acude a escuelas privadas; frente al $66 \%$ de los estudiantes con mayores recursos. Entre los países participantes en el estudio, Brasil y Argentina son los que presentan mayor diferencia social entre los estudiantes que acuden a las escuelas públicas y quiénes acuden a las escuelas privadas. Por su parte, Chile, Colombia y Ecuador presentan menores diferencias sociales entre los estudiantes.

Otro interesante trabajo es el realizado para Argentina por Gasparini y otros (2011). Los autores documentan y analizan la segregación escolar entre los estudiantes de distintos estratos socioeconómicos que asisten a escuelas públicas y privadas desde el año 1986. De acuerdo con sus resultados, el grado de segregación escolar por nivel socioeconómico muestra una tendencia alcista desde mediados de los años 1980 hasta la actualidad que se encuentra en valores moderados (índice de Disimilitud de 0,34). Frente al año 2003 en el que el $23,1 \%$ de los alumnos pobres deben pasar al sector educativo privado para que los grupos de pobres y no pobres estén igualmente distribuidos entre escuelas públicas y privadas; en el año 2009 ese porcentaje asciende al 29,3\%. Además, el índice de Aislamiento indica que mientras que en el año 2003 la probabilidad de un pobre de encontrarse con otro pobre en el mismo sector era de 0,33 , para el año 2009 está probabilidad asciende a 0,38. 
Muy interesante es el reciente estudio elaborado por Arcidiácono y otros (2014) centrado en analizar la evolución de la segregación escolar público-privado por nivel socioeconómico en América Latina. Sus conclusiones apuntan a que, con carácter general, en estas últimas dos décadas ha aumentado esta segregación público-privada en América Latina, tanto en educación primaria como en secundaria. La limitación de este trabajo es que no cuenta con datos de las escuelas donde estudian los estudiantes, por lo que es necesario seguir investigado en el tema.

Con esos antecedentes en esta investigación nos planteamos los siguientes objetivos:

1. Explorar la relación entre el porcentaje de estudiantes matriculados en escuelas públicas de Educación Primaria y la segregación escolar socioeconómica en los países de América Latina.

2. Estimar la magnitud de la segregación escolar de los subsistemas de educación pública y educación privada en cada país de América Latina

\section{Método}

Para dar respuesta a este doble objetivo se realiza una explotación secundaria de los datos del Tercer Estudio Regional Comparativo y Explicativo (TERCE) de la UNESCO. El propósito del TERCE es describir qué y cuánto aprenden los estudiantes latinoamericanos de $3^{\circ}$ y $6^{\circ}$ de Primaria en las áreas curriculares de Matemática, Lengua (lectura y escritura) y Ciencias Naturales. En línea con otras evaluaciones internacionales como PISA, el TERCE también obtuvo información de factores asociados al aprendizaje que pudieran contextualizar y hasta cierto punto explicar los resultados académicos. Por ello, junto con las pruebas de rendimiento se aplicaron cuestionarios de contexto a estudiantes, familias y docentes. Para esta investigación se seleccionan algunas variables obtenidas por el TERCE y se analizan para dar respuesta a los objetivos de investigación.

Para la selección de la muestra, en el estudio TERCE se realizó un diseño muestral estratificado, por conglomerados y bi-etápico (UNESCO, 2013). Dado que la probabilidad de selección de escuelas en la muestra propuesta es proporcional al tamaño, la probabilidad de selección de una escuela muestreada queda definida por el indicador de tamaño propuesto, es decir, matrícula de tercero, en el caso de los alumnos seleccionados en la muestra de tercer curso, y la matrícula de sexto, en el caso de los alumnos seleccionados en la muestra de sexto curso. Para el segundo nivel de selección (aula), se selecciona aleatoriamente un aula completa, lo que implica igual probabilidad de elección para cada aula dentro de la escuela. Para el tercer nivel (alumnos), el peso muestral está basado en una probabilidad de selección igual a uno. 
Con todo ello, la muestra de estudio está compuesta por total de 119.967 estudiantes de 5.733 escuelas de 15 países latinoamericanos (Argentina, Brasil, Chile, Colombia, Costa Rica, República Dominicana, Ecuador, Guatemala, Honduras, México, Nicaragua, Panamá, Paraguay, Perú y Uruguay) (Tabla 1).

Se utilizan dos variables, una de estudiante y otra de escuela. La variable de estudiante es el índice socioeconómico y cultural de la familia del estudiante (ISECF); factor calculado por el propio TERCE y obtenido a partir de los niveles educativos y ocupacionales de los padres, volumen de ingresos económicos familiares, características de la vivienda y disponibilidad de libros en el hogar. Índice estandarizado para la región y posteriormente recodificado en Cuartiles para cada país.

Además, como variable de escuela se utiliza la titularidad de la escuela: si el centro es de titularidad pública o privada. Variable dummy. Esta información procede de los criterios de muestreo utilizados en el TERCE. Hay que destacar que el TERCE no ofrece información del tipo de gestión, ni del financiamiento de las escuelas; de esta forma solo hay información de la titularidad, no de su gestión

\section{Tabla 1}

Muestra y sus características.

\begin{tabular}{l|c|c|c|c}
\hline & No de escuelas & No estudiantes & $\begin{array}{c}\text { \% en escuelas } \\
\text { públicas }\end{array}$ & $\begin{array}{c}\text { ISECF } \\
\text { promedio }\end{array}$ \\
\hline Argentina & 415 & 8.072 & 75,53 & 0,43 \\
\hline Brasil & 302 & 7.503 & 72,45 & 0,28 \\
\hline Chile & 393 & 10.089 & 37,45 & 0,83 \\
\hline Colombia & 307 & 8.528 & 56,13 & 0,14 \\
\hline Costa Rica & 393 & 7.055 & 82,23 & 0,48 \\
\hline Rep. Dominicana & 365 & 7.561 & 72,73 & $-0,28$ \\
\hline Ecuador & 391 & 9.601 & 72,01 & $-0,15$ \\
\hline Guatemala & 359 & 8.507 & 77,76 & $-0,52$ \\
\hline Honduras & 407 & 7.883 & 65,11 & $-0,46$ \\
\hline México & 336 & 7.368 & 73,10 & 0,19 \\
\hline Nicaragua & 380 & 7.805 & 73,47 & $-0,75$ \\
\hline Panamá & 372 & 7.406 & 69,46 & $-0,08$ \\
\hline Paraguay & 398 & 6.869 & 62,57 & 0,01 \\
\hline Perú & 577 & 9.956 & 67,88 & $-0,45$ \\
\hline Uruguay & 338 & 5.764 & 81,80 & 0,65 \\
\hline Total & 5.733 & 119.967 & 69,31 & 0,00 \\
\hline
\end{tabular}

Fuente: Elaboración propia a partir de los datos del TERCE.

ISECF: Índice socioeconómico y cultural de las familias de los estudiantes. 
o financiación. Ese hecho es una limitación para este estudio que más adelante será analizada.

Para estimar la segregación escolar de carácter socioeconómico en los diferentes países de América Latina se utilizan tres índices de información complementaria: El índice de Disimilitud (D), el índice de Inclusión Socioeconómica (IS) y el índice de Raíz Cuadrada $(\mathrm{H})^{1}$.

El índice de Disimilitud, o índice de Duncan (DUNCAN; DUNCAN, 1955), estima la segregación calculando la proporción de estudiantes del grupo minoritario que deberían cambiarse de escuela, sin ser reemplazados por otros estudiantes, para que la segregación sea nula, es decir, para que todas las escuelas del país tengan la misma proporción de estudiantes del grupo mayoritario y minoritario. Sus valores van de 0 a 1 y se considera que la segregación es baja si $\mathrm{D}$ toma valores entre 0 y 0,3 ; moderada si está entre 0,3 y 0,6 ; y alta cuando es superior a 0,6 (MASSEY; DENTON, 1989). Glaeser y Vigdor (2001) hablan de hipersegregación cuando D supera el 0,6 . Se ha optado por este índice por ser el más común entre los investigadores.

Matemáticamente se expresan de la siguiente forma:

$$
D=\frac{1}{2} \sum_{i=1}^{k}\left|\frac{\mathrm{x}_{1 i}}{X_{1}}-\frac{\mathrm{x}_{2 i}}{X_{2}}\right|
$$

Donde para cara país,

$\mathrm{x}_{1 \mathrm{i}} \mathrm{y}_{2 \mathrm{i}}$ son el número de estudiantes del grupo minoritario y minoritario en la escuela i;

$\mathrm{X}_{1} \mathrm{y}_{2}$ son el número total de estudiantes del grupo minoritario y mayoritario en todas las escuelas del país.

Para calcular este índice optaremos por considerar como grupo minoritario el 25\% de los estudiantes con menos ISECF (el Q1), opción habitual, pero también para el 25\% con mayor ISECF (Q4).

El segundo índice utilizado es el de Inclusión Socioeconómica, obtenido a partir del Coeficiente de Correlación Intraclase (CCI) del modelo multinivel de dos niveles (estudiante, escuela) con la variable nivel socioeconómico y cultural de las familias (ISECF) como variable dependiente en el modelo nulo. Se trata de una alternativa poco explorada hasta el momento, pero con una gran potencialidad (MURILLO, 2016; OCDE, 2010). Este índice mide la proporción de varianza del nivel socioeconómico y cultural de las familias de los estudiantes explicado por la variación entre escuelas. Concretamente, el CCI se calcula como la varianza en- 
tre-escuelas dividida por la varianza total del nivel socioeconómico y cultural de las familias de los estudiantes entre escuelas (la varianza intraescuela mas la inter-escuela). De esta forma, en un sistema con segregación escolar nula la varianza explicada por la escuela será 0 , mientras que en un sistema de máxima segregación será de 1 . Su ventaja es que no necesita dicotomizar la variable nivel socioeconómico y cultural de las familias de los estudiantes (ISECF) como si lo requieren el resto de índices, con lo que se optimiza más la información. Para su cálculo se ha utilizado el software MLwiN. Matemáticamente se expresa de la siguiente forma. Del modelo multinivel:

$\operatorname{ISECF}_{\mathrm{ij}}=\beta_{0}+\mu_{\mathrm{j}}+\varepsilon_{\mathrm{ij}}$

Con: $\left[\varepsilon_{\mathrm{ij}}\right] \sim \mathrm{N}\left(0, \Omega_{\varepsilon}\right): \Omega_{\varepsilon}=\left[\sigma_{\varepsilon}^{2}\right]$

$\left[\mu_{j}\right]-\mathrm{N}\left(0, \Omega_{\mu}\right): \Omega_{\mu}=\left[\sigma_{\mu}^{2}\right]$

Se estima el índice de Correlación Intraclase (CCI):

$$
\rho=\frac{\sigma_{\mu}^{2}}{\sigma_{\varepsilon}^{2}+\sigma_{\mu}^{2}}
$$

Por último se usa el índice de Raíz Cuadrada o de Hutchens (H) (HUTCHENS, 2004). Este índice se define como la suma, para todas las escuelas, de alejamiento de cada escuela de la igualdad distributiva. Su rango varía entre 0 y 1 , pero los valores que ofrece son menores que otros índices. Su gran virtud es que es el único que tiene la propiedad de la descomposición aditiva. Es decir, permite descomponer el índice entre subsistemas, en este caso escuelas públicas y privadas. Expresado matemáticamente es:

$$
H=\sum_{i=1}^{k}\left(\left(\frac{\mathrm{x}_{1 I}}{X_{1}}\right)-\sqrt{\frac{\mathrm{x}_{2 i}}{X_{2}} \frac{\mathrm{x}_{1 i}}{X_{1}}}\right)
$$

Por la propiedad de descomposición aditiva de esta ecuación puede ser escrita como:

$H=H_{\text {intra }}+H_{\text {entre }}$

Donde: $H_{\text {intra }}=\sum_{g=1}^{G} w_{g} H_{g} ; \operatorname{con} w_{g}=\left(P_{g} / P\right)^{1 / 2}\left(R_{g} / R\right)^{1 / 2}$

$\mathrm{G}=1, \ldots, \mathrm{G}$ subgrupos, 
$\mathrm{w}_{\mathrm{g}}$ el peso del subgrupo $g$, Pg y $\mathrm{Rg}$ el número de estudiantes en el subgrupo $g$ con respecto al grupo minoritario y mayoritario.

$\mathrm{x}_{1 \mathrm{i}} \mathrm{y} \mathrm{x}_{2 \mathrm{i}}$, representan el número de alumnos del grupo minoritario y mayoritario en la escuela $i$,

$\mathrm{X}_{1} \mathrm{y}_{2}$ son el número total de estudiantes del grupo minoritario y mayoritario en todas las escuelas del país.

Para estimar este índice también se considera como grupo minoritario tanto el 25\% de los estudiantes con menor ISECF (el Q1) como el 25\% con mayor ISECF (Q4).

\section{Resultados}

Los hallazgos de esta investigación se organizan en función del objetivo al que dan respuesta. Así, en primer lugar, se aborda la relación entre el peso de la educación privada y la segregación escolar socioeconómica. Para ello se estima la magnitud de la segregación escolar por nivel socioeconómico con los índices de Disimilitud (D), Raíz Cuadrada (H) e Inclusión Socioeconómica (IS) y, posteriormente, se relacionan con el porcentaje de estudiantes que asisten a escuelas privadas.

En segundo término, interesa conocer la segregación de los subsistemas de educación pública y educación privada en cada país de América Latina. Para ello se utilizan dos estrategias: por una parte, se estima el índice $\mathrm{H}$ en los subsistemas de educación pública y educación privada y, por otra, se analiza la composición del nivel socioeconómico de los estudiantes de las escuelas públicas y privadas en cada país.

\section{Segregación escolar socioeconómica y educación privada}

La estimación de la magnitud de la segregación escolar por nivel socioeconómico en América Latina muestra cifras globales muy altas. Así, como promedio para la Región, el 60\% de los estudiantes del cuartil con menor nivel socioeconómico debería cambiar de escuela para que no hubiera segregación (índice de Disimilitud), el 61\% de la varianza en nivel socioeconómico se da dentro de las escuelas (índice de Inclusión Socioeconómica) y el promedio de la suma de las distancia de cada escuela a la igualdad distributiva es del 0,33 (índice de Raíz Cuadrada) (Tabla 2). Como no puede ser de otra forma, esos promedios esconden grandes variaciones entre países. 
Para el índice de Disimilitud, en primer lugar, se ha optado por hacer dos estimaciones considerando como grupo minoritario, el $25 \%$ de la población de estudiantes con menor índice socio-económico y cultural de las familias (ISECF), el Q1; y el 25\% con mayor ISECF, el Q4 (Gráfico 1). Los resultados para este índice nos permiten hablar de tres tipos de países:

- Países con sistemas educativos hipersegregados (con puntuaciones superiores a 0,6): Honduras, México, Colombia y Perú (los cuatro con $0,67)$, Chile $(0,64)$, Paraguay $(0,62)$ y Brasil $(0,61)$.

- $\quad$ Países con una segregación media-alta (entre 0,5 y 0,6): Ecuador $(0,58)$, Nicaragua $(0,56)$, Argentina $(0,56)$, Costa Rica $(0,55)$, Uruguay $(0,53)$ y Guatemala $(0,52)$.

- Países con una segregación media (menos de 0,5): República Dominicana $(0,48)$.

Tabla 2

Segregación escolar socioeconómica en América Latina. Índice de Disimilitud para Q1 y Q4 en ISECF y promedio e índice de Inclusión Socioeconómica, por país.

\begin{tabular}{l|c|c|c|c}
\hline \multirow{2}{*}{} & \multicolumn{3}{|c|}{ Índice de Disimilitud } & $\begin{array}{c}\text { Índice de } \\
\text { Inclusión } \\
\text { Socioeconómica }\end{array}$ \\
\cline { 2 - 4 } Argentina & 0,5444 & 0,5721 & 0,5583 & 0,5060 \\
\hline Brasil & 0,5881 & 0,6240 & 0,6061 & 0,5961 \\
\hline Chile & 0,5651 & 0,7228 & 0,6440 & 0,5979 \\
\hline Colombia & 0,6463 & 0,6935 & 0,6699 & 0,5629 \\
\hline Costa Rica & 0,5070 & 0,6054 & 0,5562 & 0,4822 \\
\hline Rep. Dominicana & 0,4242 & 0,5359 & 0,4801 & 0,3565 \\
\hline Ecuador & 0,5371 & 0,6330 & 0,5851 & 0,5114 \\
\hline Guatemala & 0,5042 & 0,5360 & 0,5201 & 0,4466 \\
\hline Honduras & 0,5811 & 0,7687 & 0,6749 & 0,6283 \\
\hline México & 0,6400 & 0,7037 & 0,6719 & 0,5593 \\
\hline Nicaragua & 0,5432 & 0,5763 & 0,5598 & 0,3992 \\
\hline Panamá & 0,6560 & 0,7255 & 0,6908 & 0,6357 \\
\hline Paraguay & 0,5887 & 0,6592 & 0,6240 & 0,4411 \\
\hline Perú & 0,6371 & 0,6970 & 0,6671 & 0,5901 \\
\hline Uruguay & 0,4824 & 0,5828 & 0,5326 & 0,3507 \\
\hline Promedio países & 0,5630 & 0,6424 & 0,6027 & 0,6155 \\
\hline
\end{tabular}

Fuente: Elaboración propia a partir de los datos del TERCE.

ISECF: Indice socioeconómico y cultural de las familias de los estudiantes. 
Sin embargo, la segregación tiene características diferentes en cada país. En Panamá, México y Colombia, la mayor segregación se da con los estudiantes del Q1 en ISECF; mientras que en Honduras, Chile y Panamá la mayor segregación se produce en los estudiantes del Q4 en ISECF.

El análisis de la segregación mediante el índice de Inclusión Socioeconómica en cada país nos ofrece resultado análogo en su panorámica general al encontrado con el índice de Disimilitud, pero con importantes diferencias en el estudio detallado por países (Gráfico 2):

- Países que suben en el ranking: Brasil, Chile y Guatemala de forma importante, y en menor medida Argentina, Ecuador, Argentina, Costa Rica y República Dominicana.

- Se mantiene en la misma posición: Panamá, Honduras y Perú.

- Baja: De forma importante Paraguay, México, y en menos magnitud Colombia, Nicaragua, Uruguay.

Para el índice de Raíz Cuadrada (Tabla 3), los resultados son análogos: México, Perú y Colombia se presentan como los más segregados y Republica Dominicana, Uruguay, Costa Rica entre los menos segregados.

\section{Gráfico 1}

Segregación escolar por nivel socioeconómico en América Latina. Índice de Disimilitud promedio por país.

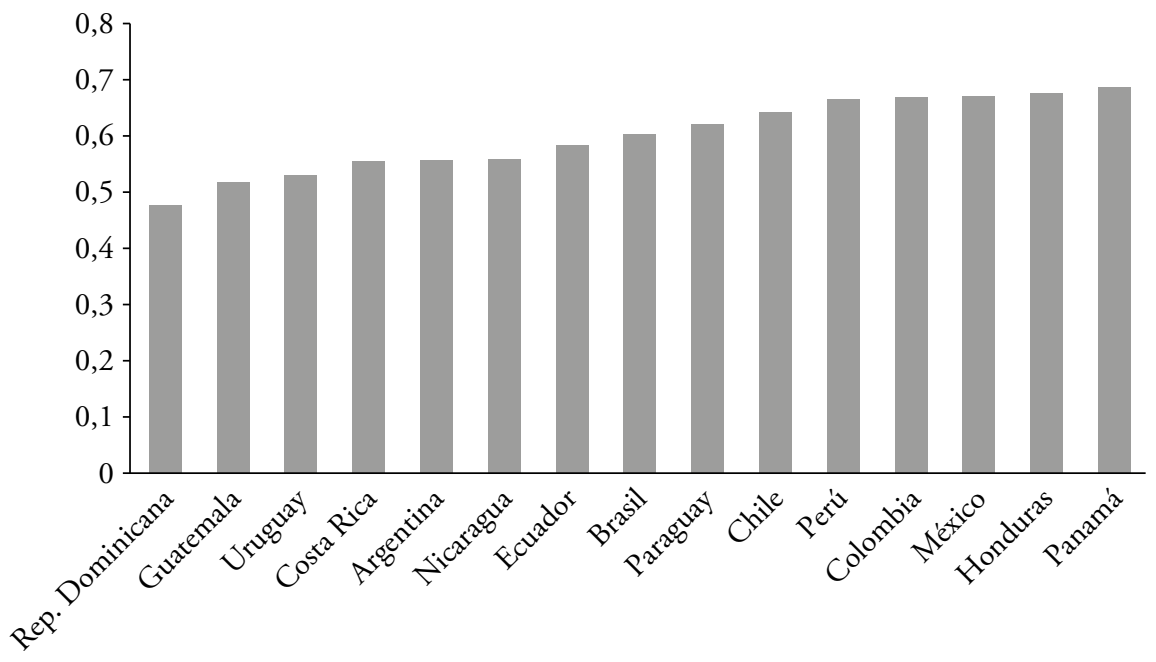

Fuente: Elaboración propia a partir de los datos del TERCE. 
No es el objetivo de esta investigación hacer una comparación de los diferentes índices, sino su relación con el porcentaje de estudiantes escolarizados en centros privados. En todo caso, la correlación entre todos los índices es muy alta, entre 0,85 (índice de Inclusión Socioeconómica e índice de Raíz Cuadrada) y 0,99 (índice de Disimilitud e índice de Raíz Cuadrada) (Tabla 4).

Estimando la relación a través del índice rho de Spearman entre el porcentaje de estudiantes matriculados en centros públicos y los diferentes índices de segregación estimados, nos encontramos una alta relación. Efectivamente, los índices de correlación entre el porcentaje de estudiantes en centros públicos y los diferentes índices de segregación son: -0,69 con el índice de Disimilitud promedio; -0,60 con el índice de Inclusión Socioeconómica y de -0,65 con el índice de Raíz Cuadrada (Tabla 3). Todas ellas estadísticamente significativas.

Estos resultados muestran una clara relación entre segregación y peso de la escuela privada en cada país. Cierto es que esta relación no puede entenderse como relación causal, pero su alta magnitud muestra un hecho incontestable.

La representación mediante un gráfico de dispersión entre el porcentaje de estudiantes en escuelas públicas y el índice de Disimilitud promedio nos muestra claramente esa relación. Así, dividido el plano en cuatro sub-planos mediante las puntuaciones promedio, el Gráfico 3 muestra que no existe ningún país con un bajo porcentaje de estudiantes en escuelas públicas y baja segregación, y prácticamente tampoco países con una alta

\section{Gráfico 2}

Segregación escolar por nivel socioeconómico en América Latina. Índice de Inclusión Socioeconómica por país.

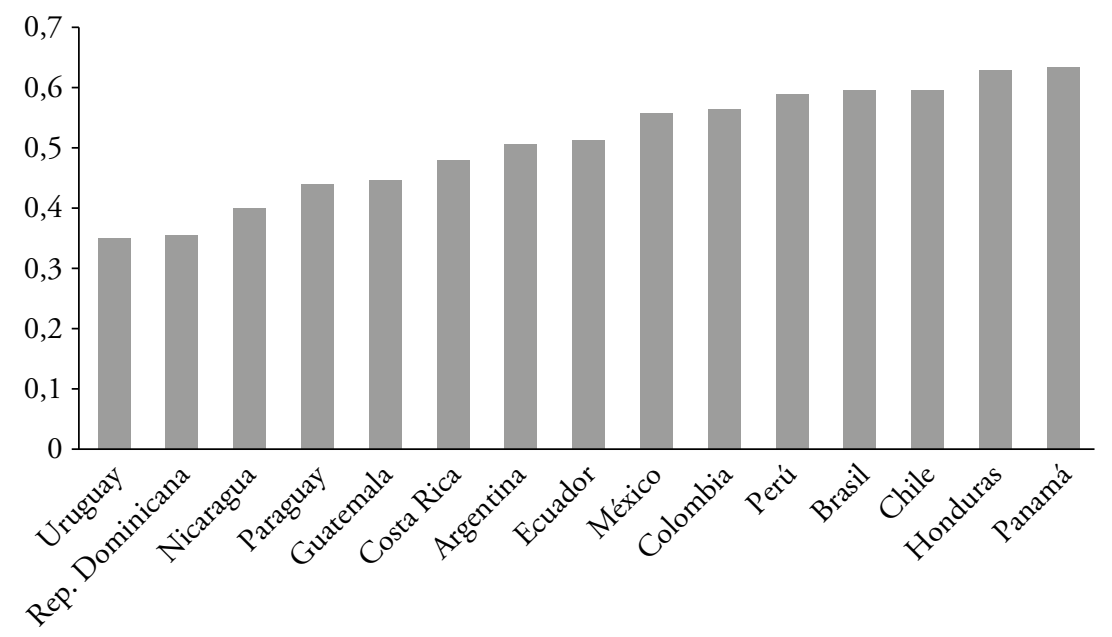

Fuente: Elaboración propia a partir de los datos del TERCE. 
proporción de estudiantes de Educación Primaria en escuelas públicas y alta segregación escolar.

Con todo ello tenemos una clara respuesta a nuestro primer objetivo: existe una fuerte relación directa entre el porcentaje de alumnos escolarizados en educación privada y la segregación de un país; relación, insistimos, que no significa necesariamente relación causal.

\section{Segregación de los subsistemas público-privado}

El objetivo planteado es determinar la segregación en los subsistemas escolares público y privado. Para ello hemos estimado el índice de Raíz Cuadrada de Hutchens; como señalamos es el único que posibilita la descomposición

\section{Tabla 3}

Segregación en escuelas públicas y privadas en América Latina.

Descomposición del índice de Raíz Cuadrada en escuelas públicas y privadas para Q1 y para Q4 en ISECF como grupos minoritarios, por país.

\begin{tabular}{l|c|c|c|c|c|c}
\hline & \multicolumn{3}{|c|}{ Para Q1 en ISECF } & \multicolumn{3}{c}{ Para Q4 en ISECF } \\
\cline { 2 - 7 } & $\begin{array}{c}\text { Todas las } \\
\text { escuelas }\end{array}$ & $\begin{array}{c}\text { Esc. } \\
\text { públicas }\end{array}$ & $\begin{array}{c}\text { Esc. } \\
\text { privadas }\end{array}$ & $\begin{array}{c}\text { Todas las } \\
\text { escuelas }\end{array}$ & $\begin{array}{c}\text { Esc. } \\
\text { públicas }\end{array}$ & $\begin{array}{c}\text { Esc. } \\
\text { privadas }\end{array}$ \\
\hline Argentina & 0,3071 & 0,2836 & 0,4376 & 0,3282 & 0,2961 & 0,1937 \\
\hline Brasil & 0,3415 & 0,2340 & 0,4936 & 0,4010 & 0,3383 & 0,2622 \\
\hline Chile & 0,3265 & 0,1389 & 0,3684 & 0,4652 & 0,4389 & 0,3628 \\
\hline Colombia & 0,4016 & 0,2211 & 0,5924 & 0,4566 & 0,4016 & 0,2986 \\
\hline Costa Rica & 0,2635 & 0,1743 & 0,6566 & 0,3744 & 0,2533 & 0,2443 \\
\hline Rep. Dominicana & 0,1978 & 0,1215 & 0,3518 & 0,2815 & 0,2165 & 0,2583 \\
\hline Ecuador & 0,2862 & 0,2062 & 0,4274 & 0,3807 & 0,3307 & 0,3194 \\
\hline Guatemala & 0,2505 & 0,1611 & 0,5153 & 0,3259 & 0,2044 & 0,3034 \\
\hline Honduras & 0,3850 & 0,2216 & 0,5831 & 0,5484 & 0,3933 & 0,2890 \\
\hline México & 0,4333 & 0,3097 & 0,6905 & 0,4605 & 0,3663 & 0,1631 \\
\hline Nicaragua & 0,2861 & 0,1937 & 0,4396 & 0,3295 & 0,2667 & 0,1967 \\
\hline Panamá & 0,4504 & 0,3020 & 0,7881 & 0,5017 & 0,3974 & 0,1740 \\
\hline Paraguay & 0,3446 & 0,2207 & 0,5076 & 0,4418 & 0,3537 & 0,3047 \\
\hline Perú & 0,4047 & 0,2653 & 0,6334 & 0,4887 & 0,4297 & 0,2814 \\
\hline Uruguay & 0,2553 & 0,2702 & 0,6773 & 0,3435 & 0,2458 & 0,1623 \\
\hline Promedio países & 0,3289 & 0,2216 & 0,5442 & 0,4085 & 0,3288 & 0,2543 \\
\hline
\end{tabular}

Fuente: Elaboración propia a partir de los datos del TERCE.

ISECF: Índice socioeconómico y cultural de las familias de los estudiantes. 


\section{Tabla 4}

Relación entre el porcentaje de estudiantes en centros públicos y diferentes índices de segregación escolar por nivel socioeconómico. Índice de correlación rho de Sperman.

\begin{tabular}{l|c|c|c|c}
\hline & $\begin{array}{c}\text { \% estudiantes } \\
\text { en centros } \\
\text { públicos }\end{array}$ & $\begin{array}{c}\text { Índice de } \\
\text { Disimilitud } \\
\text { promedio }\end{array}$ & $\begin{array}{c}\text { Índice de } \\
\text { Inclusión } \\
\text { Socioeconómica }\end{array}$ & $\begin{array}{c}\text { Índice de Raíz } \\
\text { Cuadrada } \\
\text { promedio }\end{array}$ \\
\hline $\begin{array}{l}\text { \% estudiantes en } \\
\text { centros públicos }\end{array}$ & 1 & $-0,686$ & $-0,4733$ & $-0,646$ \\
\hline $\begin{array}{l}\text { Índice de Disimilitud } \\
\text { promedio }\end{array}$ & $-0,686$ & 1 & 0,8534 & 0,982 \\
\hline $\begin{array}{l}\text { Índice de Inclusión } \\
\text { Socioeconómica }\end{array}$ & $-0,600$ & 0,818 & 1 & 0,843 \\
\hline $\begin{array}{l}\text { Indice de Raíz } \\
\text { Cuadrada promedio }\end{array}$ & $-0,646$ & 0,982 & 0,843 & 1 \\
\hline
\end{tabular}

Fuente: Elaboración propia a partir de los datos del TERCE.

Todas las correlaciones son estadísticamente significativas a un $\alpha=0,05$.

\section{Gráfico 3}

Relación entre Porcentaje de escuelas públicas y el índice D promedio.

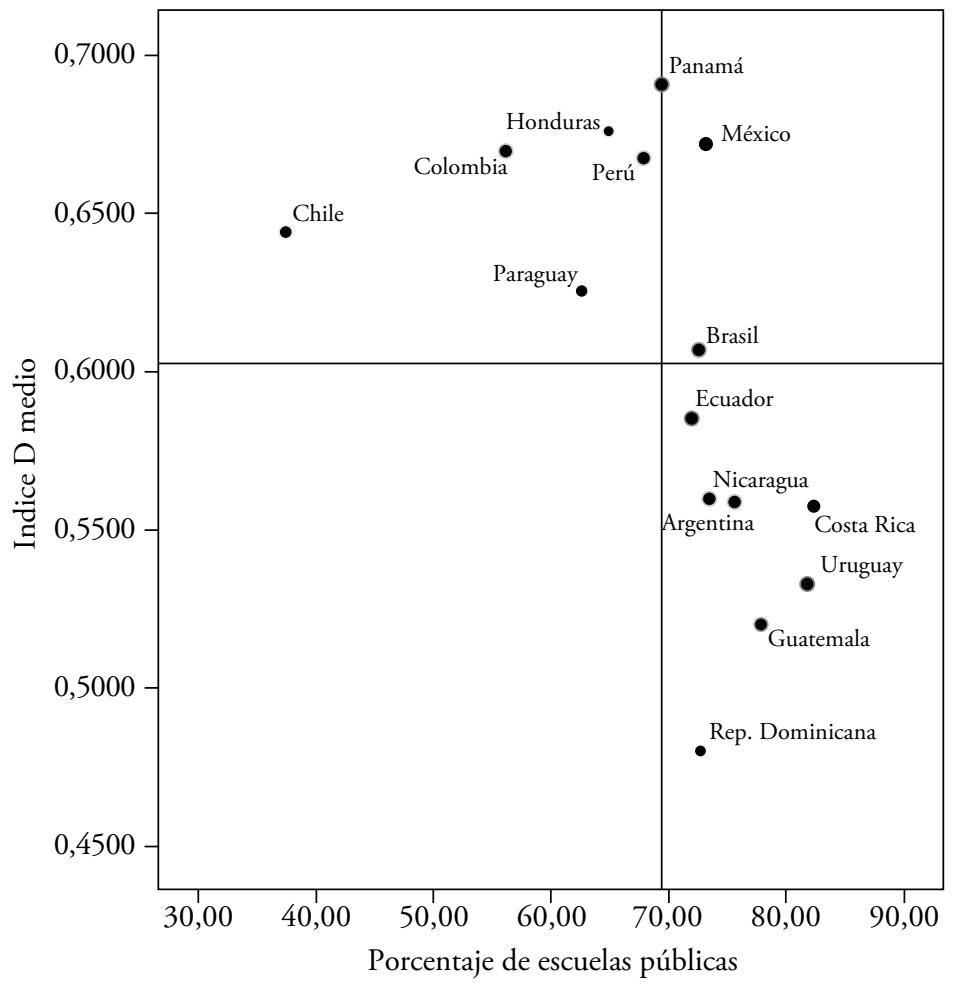

Fuente: Elaboración propia a partir de los datos del TERCE. 
aditiva del mismo y nos aporta información separada de la segregación entre los subsistemas, en este caso el subsistema de educación pública y de educación privada. El mismo, recordemos, estima la suma del alejamiento de cada escuela de la igualdad distributiva; es decir, la distancia entre la media geométrica de las participaciones de alumnos de distinto nivel socioeconómico en ausencia de segregación y la media geométrica de las participaciones reales. Y sus valores son siempre más bajos que los estimados por otros índices como el de Disimilitud o el de Inclusión Socioeconómica. De nuevo hemos estimado dicho índice con el Q1 en ISECF como grupo minoritario y con el Q4, de esta forma obtenemos una imagen más completa. Los resultados detallados se muestran en las Tablas 2 y 3 .

Analizando, en primer lugar, la descomposición de la segregación escolar público-privado para el $25 \%$ de los estudiantes con menos ISECF, encontramos que en todos los países, sin excepción, la segregación de las escuelas privadas es mucho más importante que las públicas, que hacen ese papel de integración. Sin embargo, no en todos los países se comportan igual. En Panamá, Costa Rica y Uruguay la brecha es más amplia, superando las cuatro décimas; mientras que en Argentina - el extremo contrario - se observa una menor distancia entre ambos subsistemas. En el Gráfico 4 se observan esas diferencias.

\section{Gráfico 4}

Brecha en la segregación escolar público-privado por nivel socioeconómico en América Latina. Diferencias entre el índice de Raíz Cuadrada de la segregación en centros privados y en centros públicos, considerando el Q1 y el Q4 en ISECF como grupos minoritarios, por país.

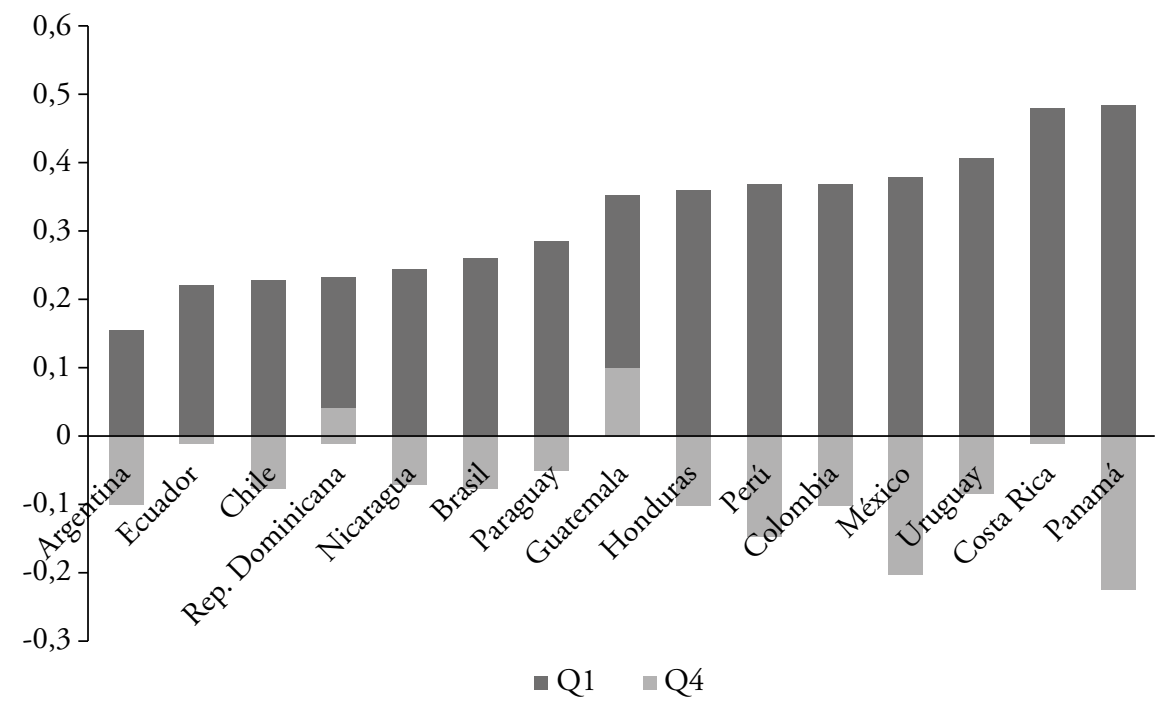

Fuente: Elaboración propia a partir de los datos del TERCE. 
La brecha de segregación público-privado para el $25 \%$ de los estudiantes con mayor ISECF (Q4), muestra un panorama radicalmente diferente. En este caso, el promedio de la segregación es mayor en las escuelas púbicas, es de magnitud mucho menor (de 0,07 puntos frente a los 0,32 para el Q1), y no todos los países siguen esta tendencia.

Efectivamente, aunque la situación más habitual es que las escuelas públicas segreguen más que las privadas para ese Q4, en Guatemala y República Dominicana, sigue siendo mayor la segregación de las escuelas privadas. En este caso, la mayor brecha se da en Panamá, México, Perú, Honduras y Argentina (todas ellas superiores a una décima) y las menores en Costa Rica y Ecuador (con una centésima de diferencia).

\section{Conclusiones}

Esta investigación buscó dar respuesta a dos objetivos. El primero de ellos es explorar la relación entre el peso de la educación privada y la segregación escolar por nivel socioeconómico en los países de América Latina en Educación Primaria. Los datos han sido claros en encontrar una fuerte relación entre ambos fenómenos: cuantos más estudiantes están escolarizados en centros de titularidad privada más segregado está el sistema educativo. Queda claro que esta relación no es necesariamente causal, seguramente una política educativa de fomento de mecanismos de cuasi-mercado escolar esté detrás de ambos fenómenos. Pero, en todo caso, se ha puesto sobre la mesa otro hecho relacionado con el fomento de la educación privada: la tendencia a una mayor segregación escolar, la existencia de sistemas más segregados e inequitativos.

Como segundo objetivo se buscaba estimar la magnitud de la segregación escolar por nivel socioeconómico de los subsistemas de educación pública y educación privada en cada país de América Latina. Los resultados indican que, para todos los países, las escuelas privadas segregan más que las públicas a los estudiantes de menor nivel socioeconómico, mientras que para los estudiantes de mayor nivel, la segregación de las escuelas públicas es mayor, pero la brecha es mucho menor y no se da en todos los países. Con ello se vuelve mostrar que la educación privada contribuye de forma determinante a la segregación escolar.

Estos resultados son coherentes tanto con estudios que estiman la segregación escolar en América Latina (por ejemplo, GASPARINI et al., 2011; MURILLO, 2016; MURILLO; MARTÍNEZ-GARRIDO, 2017a; VÁZQUEZ, 2012), así como con el trabajo de Arcidiácono y otros (2014) que estudia la segregación público y privado en América Latina. Sin embargo ha aportado datos actuales que hasta ahora no se disponían.

Nuestros resultados reflejan el contraste en torno a la magnitud de la segregación escolar por nivel socioeconómico en América Latina con respecto a otros lugares del mundo. De acuerdo con Vázquez (2012) el nivel de segregación 
por nivel socioeconómico en Estados Unidos es 0,41, algo menor en Alemania y Reino Unido (0,37 y 0,36, respectivamente). En España, de acuerdo con Murillo y Martínez-Garrido (2017b) el 38\% de los estudiantes deberían cambiarse de centro para que no hubiera segregación por nivel socioeconómico. Valores que contrastan con los resultados aportados por la presente investigación que señalan que el valor promedio de la segregación por nivel socioeconómico en América Latina es 0,60 para el conjunto de los países estudiados (valores obtenidos a través del índice de Disimilitud).

Este estudio tiene como principal virtud metodológica el uso de una base de datos con información amplia y actual, que dota a sus resultados de un interés notable. En este estudio se utilizan datos de 120.000 estudiantes y 5.700 escuelas, recogidos con un muestreo de calidad lo que hace que tengan una alta fiabilidad. También es de destacar el uso de índices poco habituales pero con un gran potencial como el de Inclusión Socioeconómica (MURILLO, 2016). Sin embargo, el trabajo tiene dos limitaciones. En primer lugar, el pequeńo número de estudiantes de la muestra en cada escuela que genera podría generar sesgos, especialmente si se trata la segregación dentro de la escuela. Pero también hay que destacar que el TERCE no recoge información de la gestión o financiación escolar; de tal forma que solo se tiene datos de si es de titularidad pública o privada, pero no de su financiación o forma de gestión. Sería deseable que en el cuarto estudio regional comparativo y explicativo del LLECE se solventaran ambas limitaciones para poder ofrecer estimaciones más precisas.

Es necesario, por tanto, seguir incorporando estudios sobre segregación escolar para el conjunto de la región y para cada uno de los países, estudios que no solo estimen su magnitud, también que analicen sus causas y consecuencias. Necesitamos tener más evidencias para mejorar la calidad y la equidad de la educación.

Con este estudio hemos querido poner sobre la mesa un elemento que sin duda puede aportar interesantes datos en el debate acerca de las implicaciones del fuerte peso de la educación privada en los sistemas educativos: sus consecuencias sobre la segregación escolar. En esta investigación hemos obtenido evidencias de que la educación privada contribuye de una forma clara a la segregación escolar. Es más, que los países que han optado por apoyar más decididamente el crecimiento del peso de la educación privada son también aquellos que tienen sistemas educativos más segregados. Seguramente no hay una relación causal, pero si hay una concepción política en la cual la equidad no se erige como prioridad máxima.

América Latina es la región más inequitativa del mundo, y los datos que tenemos indican que sus sistemas educativos se encuentran entre los más segregados. Y la relación entre ambos fenómenos no es azarosa. Difícilmente podemos contribuir a crear una sociedad más justa, sino empezamos por construir sistemas educativos más equitativos. 


\section{Notas}

1. Pueden consultarse las potencialidades y limitaciones de los índices de segregación utilizados en Murillo (2016).

\section{Referencias}

ALEGRE CANOSA, M.A. Casi-mercados, segregación escolar y desigualdad educativa: una trilogía con final abierto. Educação \& Sociedade, Campinas, v. 31, n. 113, p. 1157-1178, 2010.

ALLEN, R.; VIGNOLES, A. What should an index of school segregation measure? Oxford Review of Education, v. 33, n. 5, p. 643-668, 2007. doi: 10.1080/03054980701366306

ARCIDIÁCONO, M. et al. La segregación escolar público-privada en América Latina. Santiago: Cepal, 2014.

ARISTIZABAL, G.; GIMÉNEZ, G.; XIMÉNEZ-DE-EMBÚN, D.P. Desigualdades educativas en América Latina, PISA 2012: causas de las diferencias en desempeño escolar entre los colegios públicos y privados. Documentos de Trabajo FCEA, n. 19, p. 1-35, 2016.

BALARIN, M. La privatización por defecto y el surgimiento de las escuelas privadas de bajo costo en el Perú. ¿Cuáles son sus consecuencias?. Revista de la Asociación de Sociología de la Educación, v. 9, n. 2, p. 181-196, 2016. doi: 10.7203/RASE.9.2.8414

BELLEI, C. Expansión de la educación privada y mejoramiento de la educación en Chile. Evaluación a partir de la evidencia. Pensamiento Educativo, v. 40, n. 1, p. 1-21, 2007.

BENAVIDES, M.; LEÓN, J.; ETESSE, M. Desigualdades educativas y segregación en el sistema educativo peruano: una mirada comparativa de las pruebas PISA 2000 y 2009. Lima: Grade, 2014.

BETTS, J.; FAIRLIE, R. Explaining ethnic, racial, and immigrant differences in private school attendance. Journal of Urban Economics, v. 50, n. 1, p. 26-51, 2001. doi: 10.1006/ juec.2000.2207

BOURDIEU, P.; PASSERON, J.C. La reproducción: elementos para una teoría del sistema de enseñanza. Barcelona: Laia, 1977.

CÁRDENAS DENHAM, S. Escuelas de doble turno en México: una estimación de diferencias asociadas con su implementación. Revista Mexicana de Investigación Educativa, v. 16, n. 50, p. 801-827, 2011.

COLEMAN, J.S. Trends in school segregation, 1968-1973. Washington, D.C.: Urban Institute, 1975.

COLEMAN, J.S.; HOFFER, T.; KILGORE, S. High school achievement: public, catholic, and private schools compared. Nueva York: Basic Books, 1982.

DUARTE, J.; BOS, M.S.; MORENO, M. ¿Enseñan mejor las escuelas privadas en América Latina?: estudio comparativo usando los resultados del SERCE. Washington, D.C.: InterAmerican Development Bank, 2010. 
DUNCAN, O.; DUNCAN, B. A methodological analysis of segregation indexes. American Sociological Review, v. 20, p. 2, p. 210-217, 1955. doi: 10.2307/2088328

DUPRIEZ, V. Methods of grouping learners at school. París: Unesco, 2010.

ELACQUA, G. The impact of school choice and public policy on segregation: evidence from Chile. International Journal of Educational Development, v. 32, n. 3, p. 444-453, 2012. doi: 10.1016/j.ijedudev.2011.08.003

FERNÁNDEZ, A.; DEL VALLE, R. Desigualdad educativa en Costa Rica: la brecha entre estudiantes de colegios públicos y privados. Análisis con los resultados de la evaluación internacional PISA. Revista de la CEPAL, v. 111, n. 3, p. 37-57, 2013. doi: $10.18356 / 38309458$-es

FRANKEL, D.M.; VOLIJ, O. Measuring school segregation. Journal of Economic Theory, v. 146, n. 1, p. 1-38, 2011. doi: 10.1016/j.jet.2010.10.008

GAMBOA, L.; WALTENBERG, F.D. Inequality of opportunity for educational achievement in Latin America: evidence from PISA 2006-2009. Economics of Education Review, v. 31, p. 694-708, 2012. doi: 10.1016/j.econedurev.2012.05.002

GASPARINI, L. C. et al. La segregación escolar en Argentina: reconstruyendo la evidencia. Buenos Aires: Cedlas, 2011.

GLAESER, E.; VIGDOR, J. Racial segregation in the 2000 census: promising news. Washington, D.C.: The Brookings Institution, 2001.

GORARD, S.; SMITH, E. An international comparison of equity in education systems. Comparative Education, v. 40, n. 1, p. 15-28, 2004. doi: 10.1080/0305006042000184863

GORARD, S.; TAYLOR, C. What is segregation? A comparison of measures in terms of 'strong' and 'weak' compositional invariance. Sociology, v. 36, n. 4, p. 875-895, 2002. doi: $10.1177 / 003803850203600405$

HANUSHEK, E.A.; WOESSMANN, L. Schooling, educational achievement, and the Latin American growth puzzle. Journal of Development Economics, v. 99, n. 2, p. 497-512, 2012. doi: 10.1016/j.jdeveco.2012.06.004

HUTCHENS, R. One measure of segregation. International Economic Review, v. 45, n. 2, p. 555-578, 2004. doi: 10.1111/j.1468-2354.2004.00136.x

JAMES, D.; TAEUBER, K. Measures of segregation. CDE Working Paper 82-16, 1982. Disponível em: <http://www.ssc.wisc.edu/cde/cdewp/82-16.pdf $>$. Acesso em: 6 dez. 2016.

JAUME, D. Un estudio sobre el incremento de la segregación escolar en Argentina. Buenos Aires: CEDLAS, 2013.

KRÜGER, N. Más allá del acceso: segregación social e inequidad en el sistema educativo argentino. Cuadernos de Economía, v. 33, n. 63, p. 513-542, 2014. doi: 10.15446/cuad. econ.v33n63.45344

. The segmentation of the Argentine education system: evidence from PISA 2009.

Regional and Sectoral Economic Studies, v. 11, n. 3, p. 41-64, 2011. 
MARDONES, P. Exclusión y sobre-concentración de la población escolar migrante bajo un modelo de segregación socio-territorial. Buenos Aires: Programa Regional de Becas Clacso, 2006.

MARTÍNEZ CASAS, R.; MULLER, L. Escolarización y trabajo en los pueblos indígenas: de la segregación educativa a la discriminación laboral en las estadísticas mexicanas. En: CONGRESO IBEROAMERICANO DE EDUCACIÓN AMBIENTAL, Morelos, oct. 2006. Anales... Morelos, oct. 2006.

MASSEY, D.; DENTON, N. Hypersegregation in U.S. metropolitan areas: black and Hispanic segregation along five dimensions. Demography, v. 26, p. 373-93, 1989. doi: $10.2307 / 2061599$

The dimensions of residential segregation. Social Forces, v. 67, p. 281-315, 1988. doi: $10.2307 / 2579183$

MURILLO, F.J. Midiendo la segregación escolar en América Latina. Un análisis metodológico utilizando el TERCE. Revista Iberoamericana sobre Calidad, Eficacia y Cambio en Educación, v. 14, n. 4, p. 33-60, 2016. doi: 10.15366/reice2016.14.4.002

MURILLO, F.J.; MARTÍNEZ-GARRIDO, C. Estimación de la magnitud de la segregación escolar en América Latina. Magis, 2017a. (en prensa).

Magnitud de la segregación escolar por nivel socioeconómico en Espańa y sus comunidades autónomas y comparación con los países de su entorno. Revista de Economía Aplicada REA, 2017b. (en prensa).

OCDE. Centros privados: ¿A quién benefician? Paris: OCDE, 2011.

PISA 2009 results: overcoming social background. Equity in learning opportunities and outcomes. París: OCDE, 2010.

ORFIELD, G.; LEE, C.M. Why segregation matters: poverty and educational inequality. Boston, MA: Harvard University Press, 2005.

ORGANIZACIÓN DE LAS NACIONES UNIDAS PARA LA EDUCACIÓN, LA CIENCIA Y LA CULTURA (UNESCO). Oficina Regional de Educación de la UNESCO para América Latina y el Caribe. Evaluación de la calidad de la educación (LLECE). Tercer Estudio Regional Comparativo y Explicativo (TERCE). Santiago: Unesco/ OREALC, 2013.

PEREYRA, A. La fragmentación de la oferta educativa en América Latina: la educación pública vs. la educación privada. Perfiles Educativos, v. 30, n. 120, p. 132-146, 2008.

RANGEL, C. How do schools and neighborhoods matter for student achievement? A study of educational inequality in Cartagena, Columbia. Springfield, IL: University of Illinois, 2006.

RANGEL, C.; LLERAS, C. Educational inequality in Colombia: family background, school quality and student achievement in Cartagena. International Studies in Sociology of Education, v. 20, n. 4, p. 291-317, 2010. doi: 10.1080/09620214.2010.530855 
RUMBERGER, R.; PALARDY, G. Does segregation still matter? The impact of student composition on academic achievement in high school. The Teachers College Record, v. 107, n. 9, p. 1999-2045, 2005.

STEPHAN, W. (Ed.). School desegregation: past, present, and future. Nueva York: Springer, 2013.

VALENZUELA, J.P.; BELLEI, C.; DE LOS RÍOS, D. Segregación escolar en Chile. En: MARTINIC, S.; ELACQUA, G. (Eds.), ¿Fin de ciclo? Cambio en la gobernanza del sistema educativo. Santiago: OREALC-Unesco, 2010. p. 98-145.

. Socioeconomic school segregation in a market-oriented educational system.

The case of Chile. Journal of Education Policy, v. 29, n. 2, p. 217-241, 2014. doi: 10.1080/02680939.2013.806995

VÁZQUEZ, E. Segregación escolar por nivel socioeconómico: midiendo el fenómeno y explorando sus determinantes. Buenos Aires: CEDLAS, 2012.

WATSON, T. Inequality and the measurement of residential segregation by income in American neighborhoods. Review of Income and Wealth, v. 55, n. 3, p. 820-844, 2009. doi: $10.3386 /$ w 14908

WILSON, W.J. The truly disadvantaged. Chicago, IL: University of Chicago Press, 1987.

WINKLER, D. Higher education in Latin America: issues of efficiency and equity. Discussion Paper 77. Washington, D.C.: Banco Mundial, 1988.

Recibido en 10 de agosto de 2016.

Aprobado en 24 de octubre de 2016. 